\title{
Links between Main Frequencies of Established Rotating Stall and Rotational Frequencies and/or Blade Passing Frequencies
}

\author{
Yeshayahou Levy, ${ }^{1}$ Asaf Modelevsky, ${ }^{1}$ Joseph Pismenny, \\ Jens Friedrichs, ${ }^{2}$ and Detlev Wulff ${ }^{2}$ \\ ${ }^{1}$ Israel Institute of Technology-Technion, Israel \\ ${ }^{2}$ Technische Universität Braunschweig, Germany \\ Correspondence should be addressed to Joseph Pismenny; aeryljp@aerodyne.technion.ac.il
}

Received 16 December 2012; Revised 27 May 2013; Accepted 10 June 2013

Academic Editor: Eric Maslen

Copyright (C) 2013 Yeshayahou Levy et al. This is an open access article distributed under the Creative Commons Attribution License, which permits unrestricted use, distribution, and reproduction in any medium, provided the original work is properly cited.

\begin{abstract}
The ratios between the main frequency of rotating stall and rotational frequency may be considered in the form of exact ratios of small natural numbers if the pressure signals in compressors during rotating stall include the rotor rotation frequency component. During rotating stall in compressors with good rotor balancing (with absence of the rotational frequency component in the frequency characteristics of pressure signals), these ratios between the main frequency of rotating stall and rotational frequency are or are not in the form of ratios of small natural numbers. The experimentally received characteristics of power spectral density of pressure signals also show the presence of components with combinations of blade passing frequency and different harmonics of main rotating stall frequency.
\end{abstract}

\section{Introduction}

Rotating stall is an undesirable and even dangerous oscillatory process in a compressor. Its important characteristic is that the phase of fluctuations of pressure on a compressor circle changes in any cross-section depending on the angle of sensor positions (see, e.g., Moore and Greitzer, 1986 [1], Greitzer and Moore, 1986, [2], Longley, 1994 and 2007, [3, 4], Day and Freeman, 1994 [5], Camp and Day, 1998, [6], Day et al., 1999, [7], Bright et al., 1999, [8], Inoue et al., 2002, [9], Bergner et al., 2006, [10], etc.).

Three basic frequencies can be found in a compressor during an established rotating stall process and at its inception: the main frequency of rotating stall, the frequency of rotor rotation, and the blade passing frequency. Besides these, there can be their harmonics and also frequencies representing combinations of these three frequencies and their harmonics.

The physical nature of all these frequencies is absolutely different.

(i) Main (basic) frequency of rotating stall is caused by dynamic characteristics of the compressor and the gas system in which the compressor is included. (ii) Existence of components with rotational frequency is caused by mechanical reasons (nonideal geometrical sizes of blades, non-ideal balance of disks and blades of the rotor, shift of the axis of rotor rotation concerning the stator axis, etc.).

(iii) Occurrence of pressure fluctuations with blade passing frequency is caused by the gas flow crossing the compressor rotor blades during rotation. These components exist even when compressor balance is ideal.

The two first frequencies are frequencies of about one order; they are usually considered and analyzed in common. Blade passing frequency (being a product of the rotor rotation frequency and the number of rotor blades) surpasses by some ten times the frequency of rotating stall. The amplitude of the pressure oscillations with the blade passing frequency is much less than the amplitude of oscillation with the main frequency of rotating stall. Therefore, in the analysis of rotating stall processes, the component with blade passing frequency, as a rule, has not been taken into consideration (see, e.g., Moore and Greitzer, 1986 [1], Greitzer and Moore, 
1986, [2], Longley, 1994 and 2007, [3, 4], Day and Freeman, 1994 [5], Camp and Day, 1997 [6], Day et al., 1999; [7], etc.).

Between the blade passing frequency and the rotational frequency there are rigid links; that is, their ratios are always equal to natural numbers (equal to the number of rotor blades in the stage). At the same time there is still no definite answer to the question: are the ratios between the main frequency of the rotating stall and the rotational frequency exact ratios of small natural numbers or not. This is connected to some experimental studies, in which these relationships are ratios of small natural numbers, and other experimental studies, in which these relationships are not ratios of small natural numbers.

It is necessary to emphasize that this question is important from the point of view of classification of oscillations during rotating stall because the kind of oscillations defines the following: the investigated rotating stall is self-oscillations (if the component with frequency of rotor rotation in pressure signals are absent) or compelled oscillations (if the component with frequency of rotor rotation in pressure signals is present). In parallel there is one more question: whether the specified ratios of small natural numbers are a sign of the existence and influence of the rotor rotation frequency component in pressure signals on the process. It is important to note that the components with blade passing frequency are internal signals for the compressor as a whole and external for the gas part of system with the compressor.

Many articles about rotating stall links between the main frequency of the rotating stall and rotational frequency of the compressor are noted. Different values of the relations between these two frequencies are given in the literature (using several names). For example, Camp and Day, 1998 [6], received "mode speed" $20 \%$, "cell speed" 40\%; Gourdain et al., 2006 [11], describe "cell speed" 40\%; Day et al, 1999, [7], describe "speed" 33\% and 44\%; Bergner et al., 2006 [10], describe "frequency of the signal" $24 \%$ and $75 \%$ of rotor frequency; Inoue et al., 2002 [9], describe "rotating speed" 33\%, 49\%, and 59\%. Ariga et al., 1987 [12], describe "angular propagation speed of rotating stall” $0.333,0.667$, and 0.909 .

According to the theory of nonlinear oscillation, these relationships may be considered in the form of ratios of small natural numbers. Thus, in the above cited cases the ratios are approximately (and in more cases are exactly) equal to ratios $1: 3,1: 4,1: 5,2: 3,2: 5,3: 4,3: 5,4: 9 ; 10: 11$, and so forth. The exact ratios mean that the rotating stall process corresponds to the nonlinear compelled oscillations in the compressor (in particular, subharmonic oscillations).

Exact ratios 1:2 and 3:7 were observed in a four-stage axial compressor (see Figures 1 and 2). In this work check of this relation by one-stage axial compressors was executed. However, the exact ratios were not received. The analysis of distinctions of signals showed that in case of four-stage compressor there was in pressure signals a component with rotational frequency, and in case of one-stage compressor this component is absent. In our opinion, the reason of it is that the compressor with smaller number of stages is better balanced. Thus, the ratios between the main frequency of rotating stall and rotational frequency may be in the form of exact ratios of small natural numbers if the pressure signals

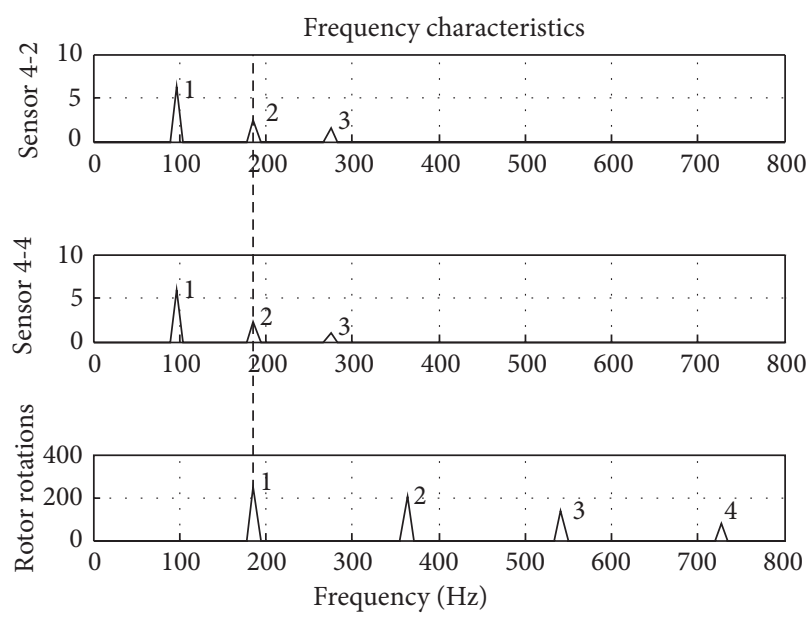

FIGURE 1: Determination of integer ratio 1:2 from frequency characteristics according to [13]. 1, 2, 3, 4-harmonics serial numbers.

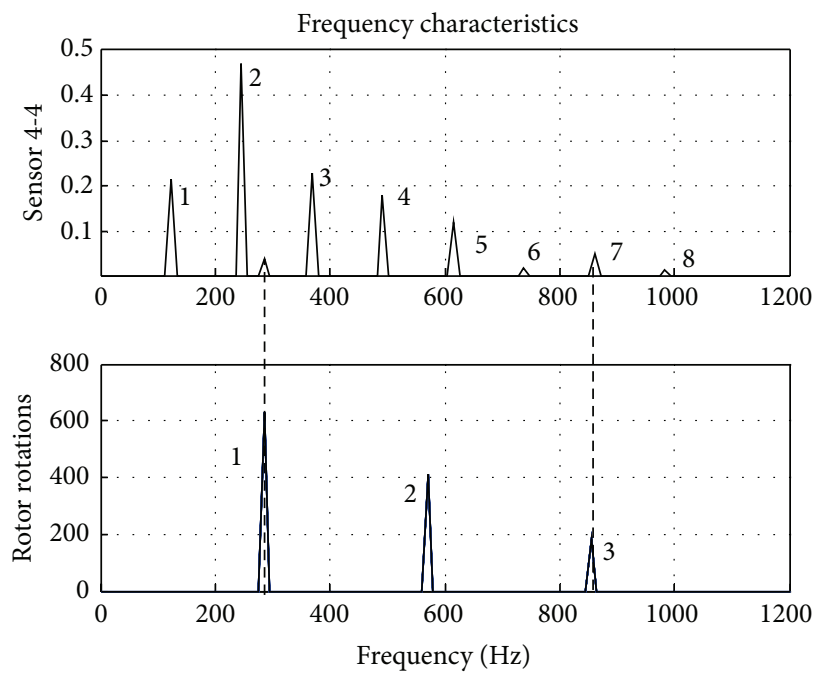

FIGURE 2: Determination of integer ratio 3:7 from frequency characteristics according to [13]. 1, 2, 3,.., 8-harmonics serial numbers.

in compressors during rotating stall include the rotational frequency component. During this research, the compressor rotor was balanced so well that the rotational frequency component in the pressure signals was practically absent. At the same time the blade passing frequency component in pressure signals was clearly visible. This allowed experimental research of multifrequency oscillations in the compressor, as in a nonlinear system in which the components of oscillations rotating stall frequency (RSF) and blade passing frequency (BPF) differed significantly both in sizes of frequencies and amplitudes. The results are presented below.

\section{Links between Parameters in Compressor in Experiments during Rotating Stall}

A diagram of links between parameters in the compressor and the measuring system during experiments is shown in 


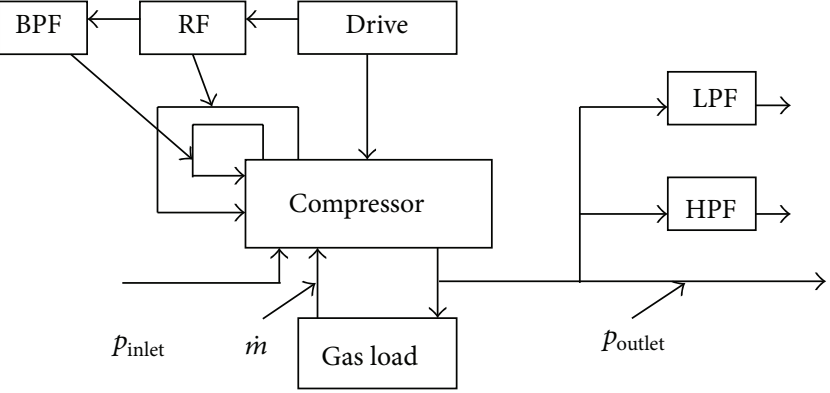

(a)

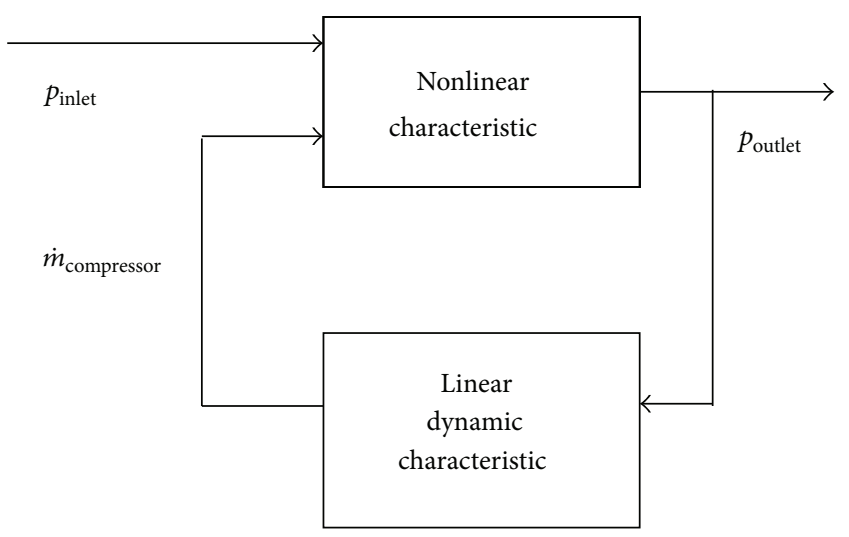

(b)

FIGURE 3: Links between parameters during rotating stall: (a) links between parameters in compressor and the measuring system in the experiments during rotating stall; (b) model of compressor as closed gas system with nonlinear and dynamic parts.

Figure 3(a). The drive provides rotation of the compressor with the RF. The rotor together with its blades creates two frequencies in the gas system: RF and BPF (in addition to the frequencies of inlet signals). The drive defines such parameters in the compressor: pressure in the compressor outlet, $p_{\text {outlet }}$, rotational frequency $(\mathrm{RF})$, and blade passing frequency (BPF). Hence, the drive influences the processes three times: once on pressure magnitude and twice on frequencies (rotational frequency and blade passing frequency).

In Figure 3(a) RF and BPF form closed contours as they are the outlet signals in the mechanical system of the compressor and simultaneously the inlet signals in the gas system of the compressor. The compressor and its gas load also form a closed contour as the load defines mass flow through the compressor, and this mass flow, in turn, together with rotational frequency defines pressure at the compressor outlet. By changing the gas load, it is possible to reduce mass flow through the compressor and receive rotating stall in the system.

The system of information processing from pressure signals defines the components with high (in the field close to $\mathrm{BPF}$ ) and low (in the field of RSF) frequencies. Thus, using the filter of low pass frequencies (LPF) and the filter of high pass frequencies (HPF), it is possible to receive and analyze the behavior of components of highfrequency and low frequency of pressure signals in the compressor separately.

For simplification we will consider only the closed contour formed by the compressor and gas load. This is a closed system with nonlinear and dynamic parts (see Figure 3(b)). Let us consider the inlet signal of the nonlinear element as a two-frequency signal:

$$
x=A_{1} \sin \omega_{1} t+A_{2} \sin \omega_{2} t
$$

We approximate the nonlinear characteristic of the compressor as a polynomial of the $n$ degree:

$$
f(x)=a_{0}+k_{1} x+k_{2} x^{2}+k_{3} x^{3}+\cdots
$$

Let us substitute (1) in (2) and consider what periodic components will take place in an outlet of the nonlinear characteristic:

$$
k_{1} x=k_{1} A_{1} \sin \omega_{1} t+k_{1} A_{2} \sin \omega_{2} t .
$$

Thus, in its outlet the component $k_{1} x$ of the nonlinear characteristic gives the components of pressure change only with frequencies at the inlet to nonlinear element $\omega_{1}, \omega_{2}$ :

$$
\begin{aligned}
k_{2} x^{2}= & k_{2}\left(A_{1} \sin _{1} \omega t+A_{2} \sin _{2} \omega t\right)^{2} \\
= & k_{2} A_{1}^{2} \sin ^{2} \omega_{1} t+2 k_{2} A_{1} A_{2} \sin \omega_{1} t \sin \omega_{2} t \\
& +k_{2} A_{2}^{2} \sin ^{2} \omega_{2} t \\
= & \frac{1}{2} k_{2} A_{1}^{2}\left(1-\cos 2 \omega_{1} t\right) \\
& +k_{2} A_{1} A_{2}\left[\cos \left(\omega_{1}-\omega_{2}\right) t-\cos \left(\omega_{1}+\omega_{2}\right) t\right] \\
& +\frac{1}{2} k_{2} A_{2}^{2}\left(1-\cos 2 \omega_{2} t\right) .
\end{aligned}
$$

Thus, the component of nonlinear characteristic $k_{2} x^{2}$ gives in its outlet the components of pressure change with frequencies $2 \omega_{1}, 2 \omega_{2}, \omega_{1} \pm \omega_{2}$ :

$$
\begin{aligned}
k_{3} x^{3}= & k_{3}\left(A_{1} \sin \omega_{1} t+A_{2} \sin \omega_{2} t\right)^{3} \\
= & k_{3} A_{1}^{3} \sin ^{3} \omega_{1} t+3 k_{3} A_{1}^{2} A_{2} \sin ^{2} \omega_{1} t \sin \omega_{2} t \\
& +3 k_{3} A_{1} A_{2}^{2} \sin \omega_{1} t \sin ^{2} \omega_{2} t+k_{3} A_{2}^{3} \sin ^{3} \omega_{2} t .
\end{aligned}
$$

This means that the component $k_{3} x^{3}$ gives components of pressure change with frequencies $\omega_{1}, 3 \omega_{1}, \omega_{2}, 3 \omega_{2}, \omega_{1} \pm$ $2 \omega_{2}, \omega_{2} \pm 2 \omega_{1}$.

Because the blade passing frequency $\omega_{\mathrm{BPF}}=\omega_{1}>0$, the basic frequency of rotating stall $\omega_{\mathrm{RSF}}=\omega_{2}>0$, and also $\omega_{1}>\omega_{2}$, the component the nonlinear characteristic $k_{3} x^{3}$ gives components of pressure change with frequencies $\omega_{1}, 3 \omega_{1}, \omega_{2}, 3 \omega_{2}, \omega_{1} \pm 2 \omega_{2}, 2 \omega_{1} \pm \omega_{2}$. 
In the general case, theoretically, the nonlinear compressor characteristic in common with change of mass flow on two frequencies gives change of pressure of these two inlet frequencies and additionally their harmonics, as well as the several linear combinations of these frequencies and harmonics:

$$
\begin{gathered}
\omega_{1}, 2 \omega_{1}, 3 \omega_{1}, \ldots, \quad \omega_{2}, 2 \omega_{2}, 3 \omega_{2}, \ldots, \\
\omega_{1} \pm \omega_{2}, \quad \omega_{1} \pm 2 \omega_{2}, \quad \omega_{1} \pm 3 \omega_{2} \ldots, \\
2 \omega_{1} \pm \omega_{2}, \quad 2 \omega_{1} \pm 2 \omega_{2}, \quad 2 \omega_{1} \pm 3 \omega_{2}, \ldots .
\end{gathered}
$$

The change of pressure with these combinations of frequencies at the inlet of the linear dynamic element (compressor connected to its gas system) also gives change of mass flow at its outlet with these combinations of frequencies. However, translation of these signals from the outlet of a nonlinear element through the dynamic part of the system will lead to change of the relationships between amplitudes of components in the spectrum and accordingly to the inlet of a nonlinear element without changing the frequency spectrum of the received signal.

The spectra of pressure oscillation during rotating stall in a concrete nonlinear system of compressor without the rotational frequency component in the pressure signals must have: (a) the blade passing frequency, (b) rotating stall frequency and also include (c) their harmonics and (d) linear combinations of all the named frequencies:

$$
\begin{aligned}
& \mathrm{BPF}, 2 \mathrm{BPF}, 3 \mathrm{BPF}, \ldots, \quad \mathrm{RSF}, 2 \mathrm{RSF}, 3 \mathrm{RSF}, \ldots, \\
& \mathrm{BPF}-\mathrm{RSF}, \quad \mathrm{BPF}-2 \mathrm{RSF}, \quad \mathrm{BPF}-3 \mathrm{RSF}, \ldots, \\
& \mathrm{BPF}+\mathrm{RSF}, \quad \mathrm{BPF}+2 \mathrm{RSF}, \quad \mathrm{BPF}+3 \mathrm{RSF}, \ldots .
\end{aligned}
$$

However, practically only part of these frequencies are actually visible in the signals of pressure oscillation on the compressor outlet during rotating stall.

Usually, in nonlinear systems such combinational frequencies, which are formed in an identical order of sizes of amplitudes and frequencies of inlet signals, are taken into consideration. In the case of rotating stall the amplitudes and frequencies of BPF and RSF differ on an order and more. Thus, we have the possibility to be convinced that combinational frequencies exist, irrespective of the relations between amplitudes and frequencies of inlet components.

\section{Experiments during Rotating Stall}

The experimental work was carried out on a single-stage lowspeed axial compressor. In our opinion, such a compressor is optimal for this research for the following reasons.

(a) In a low-speed compressor, BPF is much lower than in a high-speed compressor. Therefore, the same frequency range (in Hertz) corresponds to a greater order of harmonic of BPF. This allows capturing more harmonics and combinatorial frequencies of them.

(b) In the frequency spectrum of a single-stage compressor there is only one BPF. Thus the overall picture

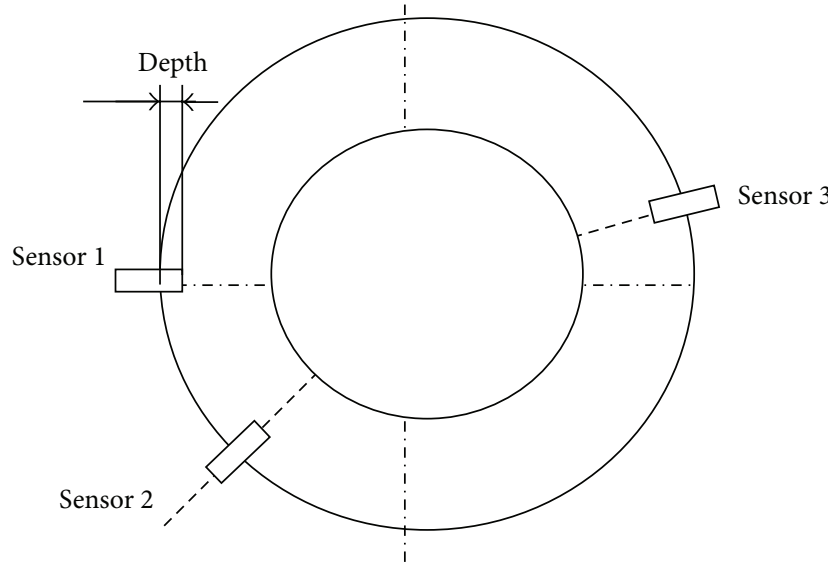

FIGURE 4: Position of sensors in one cross-section of compressor.

of a spectrum of oscillations does not become complicated because of oscillation interference on two or more BPF (as in two or more stage compressors).

The present study was performed on single-stage lowspeed axial compressor belonging to the Institut für Flugantriebe und Strömungsmaschinen, Technische Universität Braunschweig. This compressor consists of a single stage (rotor and stator) with an outlet diameter of $400 \mathrm{~mm}$ while different blade designs and two different Hub/Tip ratios of $v=0,55$ and $v=0,70$ where investigated (with height of blades $60 \mathrm{~mm}$ and $90 \mathrm{~mm}$ resp.). The different designs are leading to different numbers of blades:

(i) Type 7143, $\nu=0,70 ; 20$ rotor and 23 stator blades;

(ii) Type 7144, $v=0,70 ; 20$ rotor and 23 stator blades;

(iii) Type 7157, $v=0,70 ; 20$ rotor and 23 stator blades;

(iv) Type 7160, $v=0,55 ; 16$ rotor and 19 stator blades.

The characteristics of the different types of blades are shown in Table 1. Table 2 summarizes the characteristics of the parameters of the compressor design according to the types of blades used. The diagram of sensor positions in one cross-section of the compressor during experiments is shown in Figure 4.

In this figure the gas flow in the compressor moves perpendicularly to the figure plane (in the annulus, between the two circles). The larger circle corresponds to the external diameter of the compressor, and the small circle corresponds to its internal diameter.

Probes are mounted on the circumference in one crosssection of the stator. The angle between sensors 1 and 2 is equal to $45^{\circ}$ and the angle between sensors 2 and 3 is equal to $150^{\circ}$. Depth of probes insertion (relative to the external diameter of the flow) can be regulated.

The rotor of compressor is driven by an electric motor. During rotating stall the speed of rotor rotation was maintained constant by the motor. Therefore during rotating stall the frequencies RF and BPF were also constant.

The probe represents a thin pipe of small diameter with pressure sensitive element at its end (Kulite XCS-062-5D) 


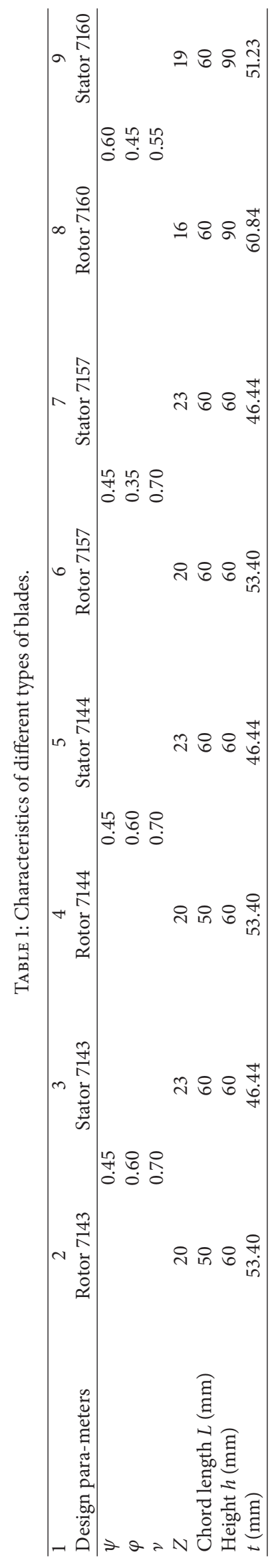




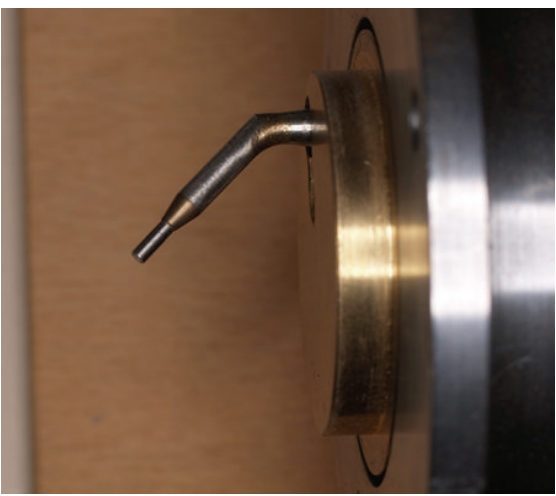

(a)

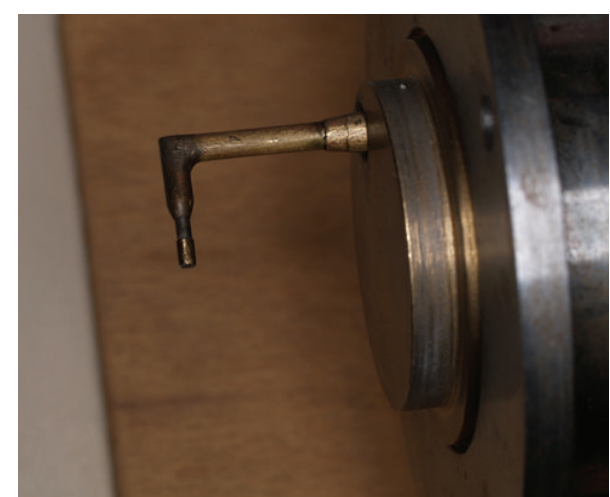

(b)

FIgURE 5: Probes of J-form (a) and L-form (b).

TABLE 2: Summary characteristics of change of parameters by used types of blades.

\begin{tabular}{lc}
\hline Hub/Tip ratio $v$ & $0.55 ; 0.7$ \\
Number of rotor blades, $z$ & $16 ; 20$ \\
Number of stator blades, $z$ & $19 ; 23$ \\
Chord length $L(\mathrm{~mm})$ & $50-60$ \\
Height of blades $h(\mathrm{~mm})$ & $60 ; 90$ \\
Height/chord $h / L$ & $0.909-1.5$ \\
Rotor speed & Variable up to $4500 \mathrm{rpm}$ \\
Rotor frequency & $75 \mathrm{~Hz} ; 70 \mathrm{~Hz} ; 66.7 \mathrm{~Hz} ; 60 \mathrm{~Hz}$ \\
Blade passing frequencies & $960-1500 \mathrm{~Hz}$
\end{tabular}

with natural frequency calculated to be $150 \mathrm{kHz}$. Hence there was no need for any corrections for the frequency response up to $20 \mathrm{kHz}$ and the signal of the pressure sensor is sensitive to the absolute pressure value as well as its fluctuations and therefore does not depend on the source of these fluctuations (fluctuation in flow rate or fluctuation of sound waves).

Mechanical imbalance of the rotor leads to fluctuations in the pressure signal due to the variations in the relative flow geometry and therefore can appear at the rotational frequency and its harmonics. These components of fluctuations can be observed both, during a steady state work of compressor and during rotating stall (see, e.g., Levy et al., [13]).

Several probes with pressure sensors on the ends of probes are mounted as shown on Figure 5. Due to this FRAP-design (fast response aerodynamic probe) the delay of transfer of pressure from the inlet probe to the pressure sensors in these probes is absent. The probes have one important difference: the probes of sensors 1 and 2 have Jform ends (short channel forming a corner $135^{\circ}$ with the basic channel); probe 3 has an L-form end (short channel perpendicular to the basic channel). Such a distinction in the design of probes allows seeing dependence of frequency spectra of signals of pressure in one cross-section of the compressor during rotating stall from the angle of installation of a sensitive element in gas flow.

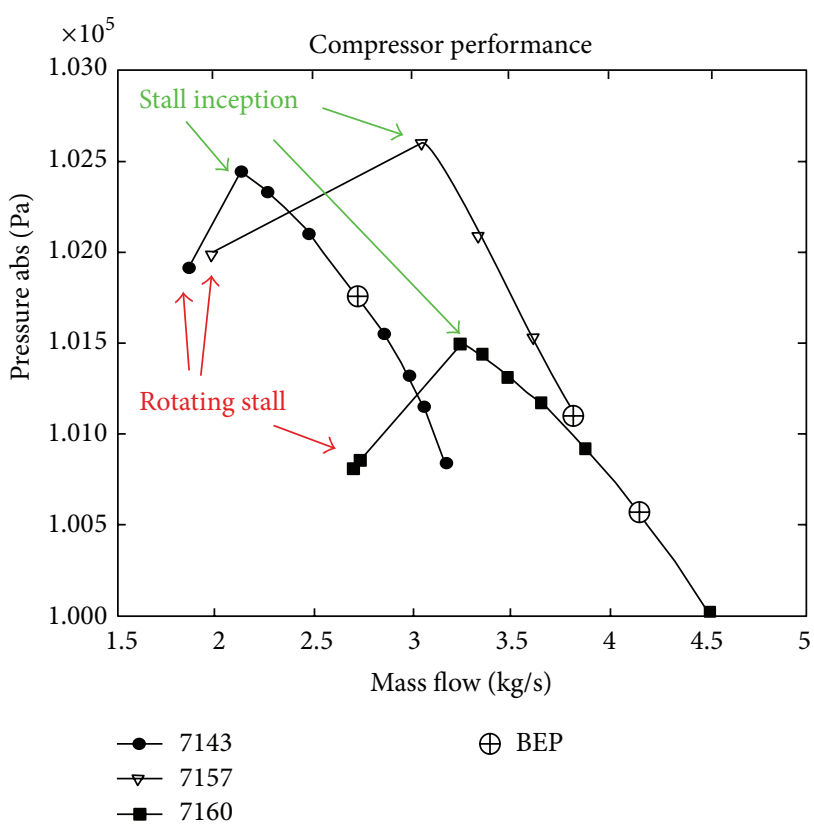

Figure 6: Performance diagram of different compressors.

In Figure 6, the performance diagrams of different investigated compressors are shown. The stall is received as a result of mass flow reduction. It can be stated that all investigated compressors will stall at mass flow range that equals to 73$80 \%$ of the value at the best efficiency points (BEP).

\section{Frequency Characteristics of Pressure Changes during Rotating Stall: Combinational Frequencies during Rotating Stall}

Typical frequency characteristics of pressure signals in onestage compressors during rotating stall are presented in Figures 7 and 8. Figure 7(a) clearly shows a component of the first harmonic of RSF (with maximal value of PSD) for all sensors as well as a much smaller second harmonic 2RSF. 

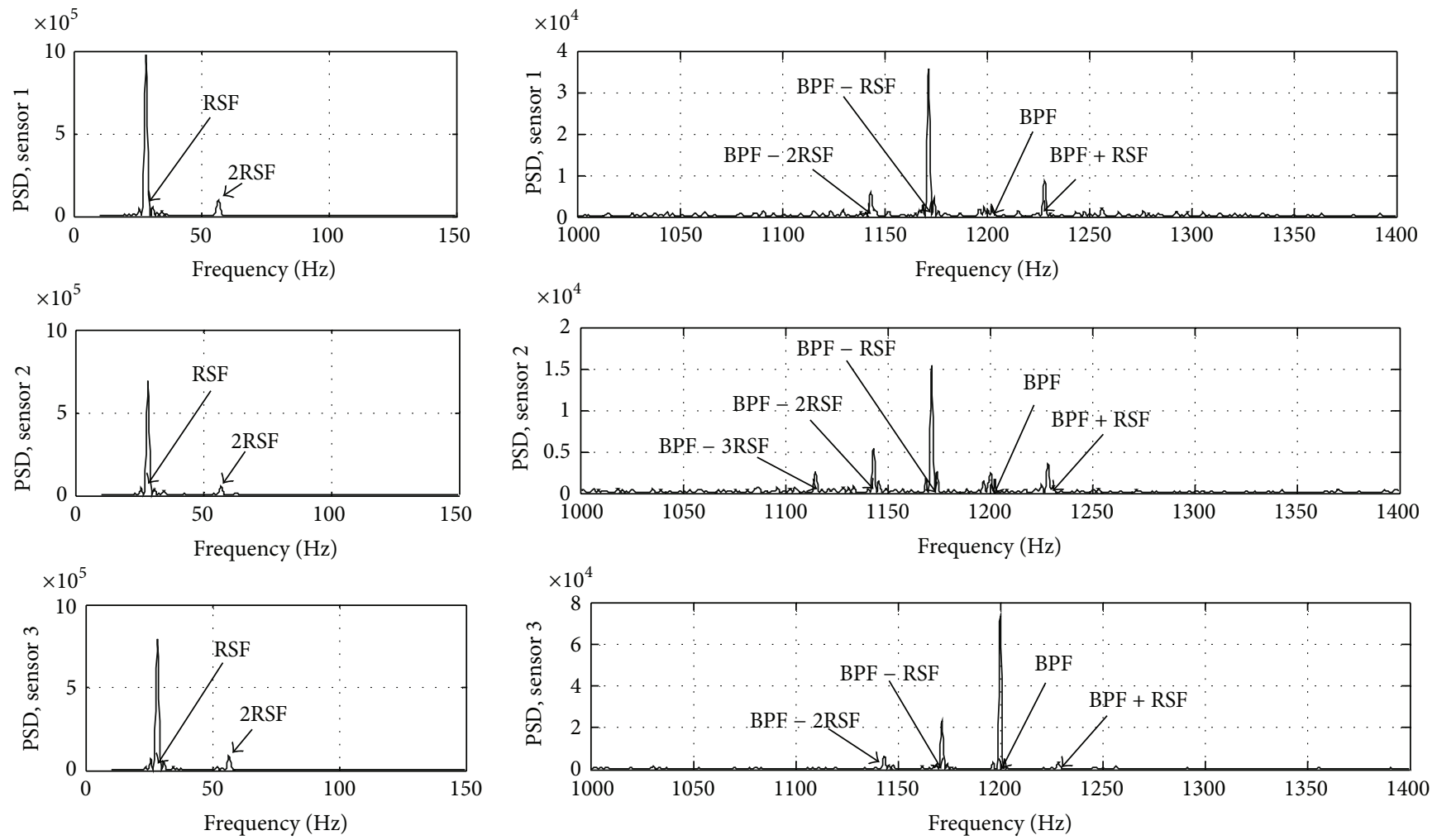

(a)

(b)

FIGURE 7: PSD characteristics of compressor with 20 rotor and 23 stator blades 7143. Frequency of rotor rotation $60 \mathrm{~Hz}$; depth of sensors $5 \mathrm{~mm}$.

The RRF component is absent in all sensors. On Figure 7(b), for all sensors, components with frequencies BPF, BPF-RSF, $\mathrm{BPF}+\mathrm{RSF}$, and also BPF - 2RSF (by all sensors), and BPF 3RSF (by sensor 2) are clearly visible. The right hand parts of the PSD characteristics of sensors 1 and 2 essentially differ from the PSD of sensor 3: by sensors 1 and 2 the greatest is the component with combinational frequency BPF - RSF. We may consider the characteristic of sensor 3 in Figure 7 as classical because the value of PSD for BPF is greater than values of PSD for combinational frequencies BPF - RSF, $\mathrm{BPF}+\mathrm{RSF}$, and so forth.

The component with rotational frequency $(\mathrm{RF})$ is practically absent in all pressure signals because of good balancing of the rotors in all the above described compressors. Therefore, its harmonics and also combinational frequencies with these frequencies are also absent. In the general case, the amplitudes of the components with combinational frequencies, including BPF, during rotating stall may be greater than the amplitudes of the components with blade passing frequency.

The left hand parts of all PSD characteristics of pressure sensor signals mounted in one cross-section of the compressor at one depth are identical. The right hand parts of PSD characteristics contain an identical spectrum of combinational frequencies in all cases BPF, BPF - RSF, $\mathrm{BPF}+\mathrm{RSF}, \mathrm{BPF}-2 \mathrm{RSF}, \mathrm{BPF}+2 \mathrm{RSF}, .$. , but amplitudes of components of different sensors on the same frequencies may differ from each other.
The frequency characteristics of pressure signals presented in Figure 8 are analogous. The frequency characteristics of sensors 1 and 2 in the field of BPF and their combinational frequencies are approximately identical. The difference between the frequency characteristics of sensors 1 , and 2 on the one hand and 3 on the other hand is due to the different direction of the inlets.

Spectra of combinational frequencies do not depend on size of corner between short and basic channels of the probes. At the same time amplitudes of fluctuations on these frequencies essentially depend on the form of the probes.

Thus, components with combinational frequencies occur at the outlet of nonlinear elements even when there are significant differences in sizes (10 time or more) of frequencies and amplitudes of components at the inlet of these elements. The frequency characteristics of signals of pressure sensors (having identical characteristics and mounted in the compressor in the same cross-section) during rotating stall can have distinctions in the right hand part of spectra because of distinction in the form of the probes.

\section{Results and Discussions}

5.1. Links between the Established Frequencies of Rotating Stall and the Frequency of Rotor Rotation. The links between rotational frequencies and main rotating stall frequencies are shown in Figure 9. The links between rotational frequencies 

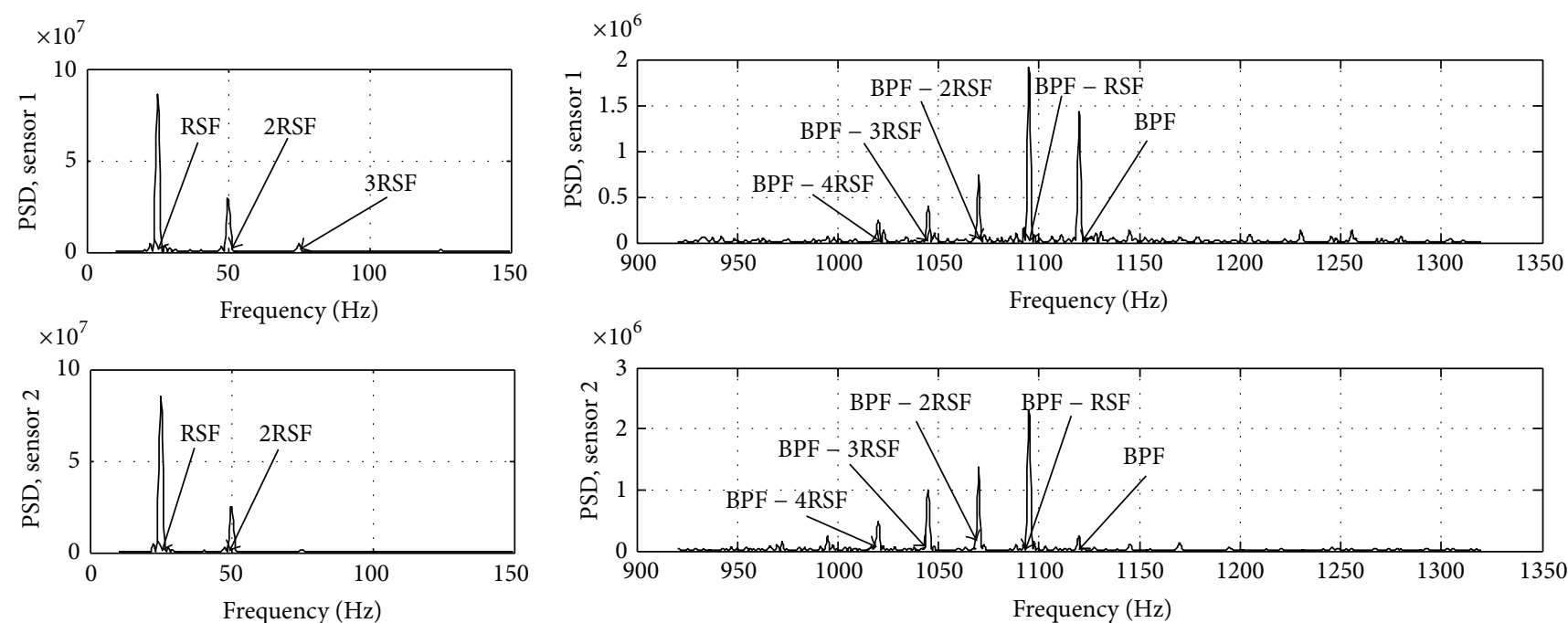

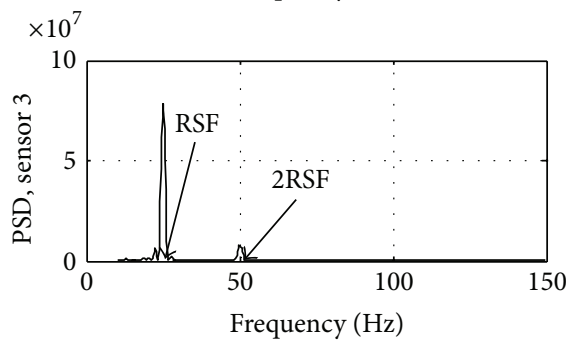

(a)

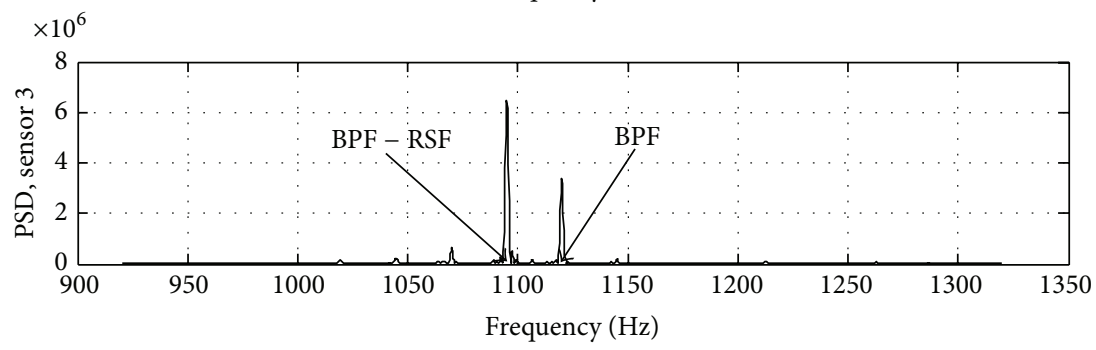

(b)

FIGURE 8: PSD characteristics of compressor with 16 rotor blades and 19 stator blades 7160 . Frequency of rotor rotation $70 \mathrm{~Hz}$; depth of sensors $5 \mathrm{~mm}$.

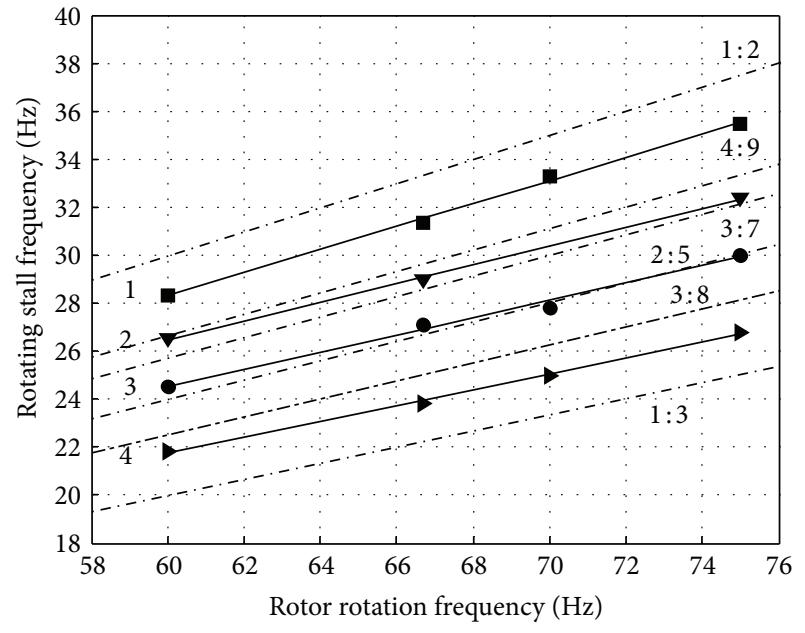

FIGURE 9: Links between rotational frequencies and main frequencies of established rotating stall.

and ratios of "main rotating stall frequencies versus rotational frequencies" are shown in Figure 10. For comparison the lines of ratios of natural numbers $1: 2,1: 3,3: 8,2: 5,3: 7$, and $4: 9$ are also shown.

The obtained characteristics are located as follows:

(i) for the compressor with 20 rotor blades and 23 stator blades 7143 (line 1)-between lines $4: 9$ and 1:2;

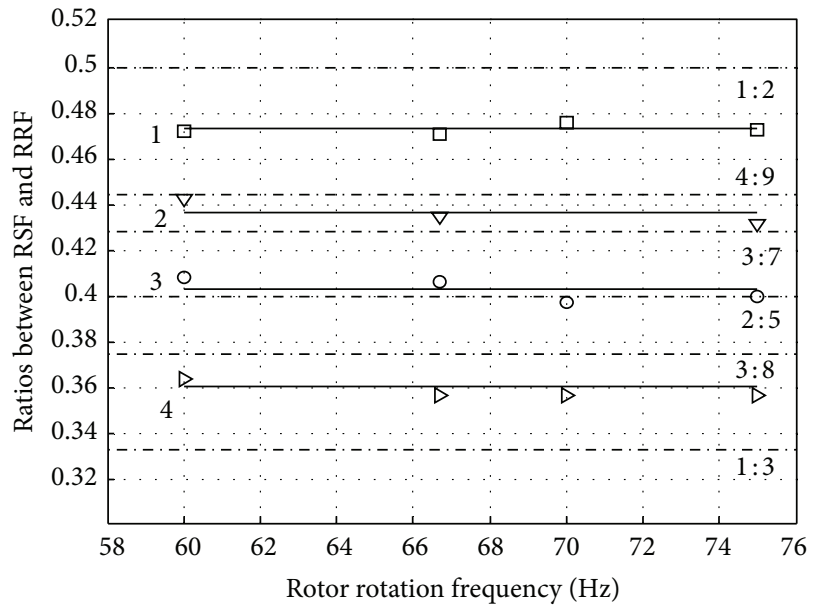

FIGURE 10: Links between rotational frequencies and ratios "main frequencies of established rotating stall to rotational frequencies."

(ii) for the compressor with 20 rotor blades and 23 stator blades 7144 (line 2) - between lines $3: 7$ and $4: 9$, close to both lines;

(iii) for the compressor with 20 rotor blades and 23 stator blades 7157 (line 3) - the characteristic is located very close to the line of $2: 5$; 
(iv) for the compressor with 16 rotor blades and 19 stator blades 7160 (line 4 )-between lines $1: 3$ and $3: 8$.

Thus, the good coincidence with a ratio of small natural numbers 2:5 takes place only for the compressor with 20 rotor blades and 23 stator blades 7157. In other cases the links between the main rotating stall frequencies and the rotational frequencies exist, however, not in the form of exact ratios of small natural numbers.

In the classical instability criteria of compressor (e.g., Moore and Greitzer, Greitzer and Moore, [1, 2]) the Helmholtz frequency can be described by speed of sound, cross-section behind the compressor, length of the compressor, and volume behind. These criteria were (a) developed for models with the concentrated parameters and (b) do not consider influence of features of geometry of blades and their quantity, the profile of channels and so forth. By means of Figures 9 and 10 it is possible to make an experimental assessment of joint influence on main frequencies of rotating stall of that fact that the real compressor is system with the distributed parameters and of various profiles of blades and their quantity.

For compressors with equal geometrical sizes, equal numbers of blades and different profiles of the blades (see on Figures 9 and 10 lines 1, 2, 3-lines for compressors with equal heights of the blades $60 \mathrm{~mm}$ ) the experimentally received ratios "main frequencies of established rotating stall to rotational frequencies" are equal to $0.4-0.475$; that is, the distinction is less than $\pm 10 \%$. In the case of a compressor with greater height of the blades $(90 \mathrm{~mm}$ by compressor with 16 rotor blades and 19 stator blades 7160) the ratios of frequencies were smaller than in cases of compressors with smaller height of the blades $(60 \mathrm{~mm}$ by all other variants of blades).

Thus, experimental confirmation of good correlation of RSF with RF (and also BPF) is received.

\subsection{Discussion about Why the Combinational Frequencies of} $R F$ and RSF Were Not Noticed Earlier. After detection of combinational frequencies in pressure signals of compressors with RSF and BPF and without the rotational frequency component, there is a natural question as to why the combinational frequencies have not been noticed earlier during rotating stall in compressors which have the RF component. To answer this question it is necessary to realize that this represents combinational frequencies only in the case of the existence of RF components (and its harmonics) and RSF (and its harmonics) in pressure signals.

In such a case, the pressure oscillation in the compressor outlet during rotating stall must have the components of combinational frequencies:

$$
m * \mathrm{RF}-n * \mathrm{RSF}, \quad m * \mathrm{RSF}+n * \mathrm{RRF},
$$

where $m=1,2,3, \ldots$ and $n=1,2,3, \ldots$.

For example, as may be seen in Figures 1 and 2 the frequency characteristics of pressure signals during rotating stall with ratios of $\omega_{\mathrm{RSF}} / \omega_{\mathrm{RF}}=3: 7$ (i.e., $3 \mathrm{RF}=7 \mathrm{RSF}$ ) and $\omega_{\mathrm{RSF}} / \omega_{\mathrm{RF}}=1: 2$ (i.e., $\mathrm{RF}=2 \mathrm{RSF}$ ). (a) In case $\omega_{\mathrm{RSF}} / \omega_{\mathrm{RF}}=1: 2$, the combinational frequencies

$$
\begin{gathered}
\mathrm{RF}-\mathrm{RSF}=\mathrm{RSF} \quad(1 \mathrm{st} \text { harmonic of RSF }), \\
\mathrm{RF}=2 \mathrm{RSF} \quad(2 \mathrm{nd} \text { harmonic of RSF }), \\
\mathrm{RF}+\mathrm{RSF}=3 \mathrm{RSF} \quad(3 \mathrm{rd} \text { harmonic of RSF }) .
\end{gathered}
$$

It is possible to see that these combinational frequencies are simultaneously also the 1,2, and 3 harmonics of RSF.

(b) In case $\omega_{\mathrm{RSF}} / \omega_{\mathrm{RF}}=3: 7$, the combinational frequencies

$$
\begin{aligned}
3 \mathrm{RF}-5 \mathrm{RSF} & =3 \mathrm{RF}-5 * \frac{3}{7} \mathrm{RF}=\frac{21-15}{7} \mathrm{RF} \\
& =2 * \frac{3}{7} \mathrm{RF}=2 \mathrm{RSF} ; \\
3 \mathrm{RF}-4 \mathrm{RSF} & =3 \mathrm{RF}-4 * \frac{3}{7} \mathrm{RF}=\frac{21-12}{7} \mathrm{RF} \\
& =3 * \frac{3}{7} \mathrm{RF}=3 \mathrm{RSF} ; \\
3 \mathrm{RF}-3 \mathrm{RSF} & =3 \mathrm{RRF}-3 * \frac{3}{7} \mathrm{RF}=\frac{21-9}{7} \mathrm{RF} \\
& =4 * \frac{3}{7} \mathrm{RF}=4 \mathrm{RSF} ; \\
3 \mathrm{RF}-2 \mathrm{RSF} & =3 \mathrm{RRF}-2 * \frac{3}{7} \mathrm{RF}=\frac{21-6}{7} \mathrm{RF} \\
& =5 * \frac{3}{7} \mathrm{RF}=5 \mathrm{RSF} ; \\
& =6 * \frac{3}{7} \mathrm{RF}=6 \mathrm{RSF} . \\
3 \mathrm{RF}-\mathrm{RSF} & =3 \mathrm{RRF}-1 * \frac{3}{7} \mathrm{RF}=\frac{21-3}{7} \mathrm{RF} \\
& =3
\end{aligned}
$$

It is possible to see these combinational frequencies are simultaneously also the 2, 3, 4, 5, and 6 harmonics of RSF. Thus, the answer to the question is as follows: the combination frequencies were also taking place earlier, but were not noticed, because in cases of existence in pressure signals of the RF component the combination frequencies coincide with the harmonics of RSF.

5.3. Correlation between the Rotational Frequency (RF), Blade Passing Frequency (BPF), and Main Rotating Stall Frequency $(R S F)$. Theoretically, it can be stated that during small changes in the RF, the compressor compression, and consequently its exit pressure, $p$, will change as well:

$$
\Delta p=k_{1} * \Delta \mathrm{RF}
$$

During pressure variations, the gas flow properties (in particular its sound speed, $a$ ) also change:

$$
\Delta a=k_{2} * \Delta p .
$$


During variations in the speed of sound, the dynamic parameters of the compressor (such as its own natural frequency, $\mathrm{OF}$ ) will also change:

$$
\Delta \mathrm{OF}=k_{3} * \Delta a
$$

One form such frequencies is the RSF:

$$
\Delta(\mathrm{RSF})=\Delta(\mathrm{OF}) .
$$

As a result, the RSF varies. At the same time also the BPF changes: $\mathrm{BPF}=\mathrm{Z}_{\mathrm{rot}} * \mathrm{RF}$ also changes:

$$
\Delta(\mathrm{RSF})=k_{1} k_{2} k_{3} * \Delta(\mathrm{RF})=K * \Delta(\mathrm{RF})
$$

where $K=k_{1} k_{2} k_{3}$ and $\Delta(\mathrm{RSF})=K / Z_{\text {rot }} * \Delta(\mathrm{BPF})$.

Hence theoretically RSF is in close correlation with RF and BPF.

These phenomena could clearly be seen in Figures 9 and 10 where linear relations where measured between the rotating stall frequencies and the frequencies of rotor rotations within a relatively large range.

\section{Conclusions}

(1) Generally the frequency characteristics of pressure signals in compressors during rotating stall include the main frequency of rotating stall, the rotational frequency, the blade passing frequency, their harmonics, and also combinational frequencies of these three frequencies and their harmonics. During rotating stall, in compressors with good rotor balancing (with the absence of the rotational frequency component in the frequency characteristics of pressure signals), there are only the components with the main frequency of rotating stall, the blade passing frequency, their harmonics, and also their combinational frequencies.

(2) Usually in the analysis of pressure signals during rotating stall, the blade passing frequency component is seldom taken into consideration because of essential distinctions of its amplitude and frequency from the amplitudes and frequencies of the two other components of rotating stall processes:

(i) Blade passing frequency (being the product of the rotor rotation frequency and the number of rotor blades) surpasses some ten times the frequencies of rotating stall and rotor rotation.

(ii) Amplitude of the component of oscillation on the blade passing frequency is ten times less than the amplitude of oscillation in the main frequency of rotating stall.

(3) The presence of links between the main frequency of rotating stall and the rotational frequency has been noted in the literature for a long time. In the case of presence in the pressure signals of components with the rotational frequencies these links can be in the form of exact ratios of small natural numbers.
(4) In the presented research the rotor of the compressor was balanced so well that the component with rotational frequency in the pressure signals was practically absent. At the same time the blade passing frequency component in pressure signals was clearly visible. This allows carrying out experimental study of multifrequency oscillations in the compressor, as in a nonlinear system where the two main components of oscillations differed importantly both in sizes of frequencies and in sizes of amplitudes.

The experimentally received characteristics of power spectral density (PSD) of pressure signals show the presence in pressure signals of earlier not described components with different combinations of blade passing frequency with main rotating stall frequency and their harmonics.

(5) The links between the main frequencies of rotating stall and the rotational frequencies also exist in cases of absence in pressure signals of rotational frequency components. However, (unlike the cases of presence of the rotational frequencies) in such cases these links are or are not in the form of exact ratios of small natural numbers.

(6) In cases of absence of components with the rotational frequencies, the ratios between the main frequencies of rotating stall and the frequencies of rotor rotation differ considerably depending on types of rotor and stator blades and their quantity and do not depend upon the rotational frequencies, at least in the experimentally checked area of $80-100 \%$ of the nominal rotational frequency.

(7) The amplitudes of the components with combinational frequencies may be greater than the amplitudes of the components with blade passing frequency.

\section{Nomenclature}

A: $\quad$ Amplitude, $\mathrm{N} / \mathrm{m}^{2}$

BEP: Best efficiency point, dimensionless

BPF: Blade passing frequency,

$\mathrm{BPF}=\mathrm{RF} * Z_{\text {rot }}, \mathrm{Hz}$

HPF: $\quad$ High pass frequencies, $\mathrm{Hz}$

LPF: $\quad$ Low pass frequencies, $\mathrm{Hz}$

PSD: $\quad$ Power spectral density, dimensionless

RF: $\quad$ Rotational frequency, $\mathrm{Hz}$

RSF: $\quad$ Main frequency of rotating stall, $\mathrm{Hz}$

$f: \quad$ Frequency, $f=1 / T, \mathrm{~Hz}$

in: $\quad$ Mass flow, $\mathrm{kg} / \mathrm{s}$

p: $\quad$ Pressure, $\mathrm{N} / \mathrm{m}^{2}$

$t: \quad$ Time, s

$t$ : Distance between blades on a circle of maximal diameter $(t=\pi \mathrm{D} / Z), \mathrm{mm}$

T: $\quad$ Period, s

$Z_{\text {rot }}$ : Number of rotor blades, dimensionless

$\omega: \quad$ Circular frequency, $1 / \mathrm{s}$. 


\section{Acknowledgments}

The authors would like to thank the Ministry of Sciences and Culture of the Germany State of Lower Saxony and the Volkswagen Foundation, Hannover, for supporting this research.

\section{References}

[1] F. K. Moore and E. M. Greitzer, "A theory of post stall transients in axial compression systems-part I: development of equations," Journal of Engineering for Gas Turbines and Power, vol. 108, no. 1, pp. 68-76, 1986.

[2] E. M. Greitzer and F. K. Moore, "A theory of post-stall transients in axial compression systems-part II: application," Journal of Engineering for Gas Turbines and Power, vol. 108, no. 2, pp. 231239, 1986.

[3] J. Longley, "A review ofnonsteady flow models for compressor stability," Journal of Turbomachinery, vol. 116, pp. 202-215, 1994.

[4] J. Longley, "Calculating stall and surge transients," in Proceedings of the ASME Turbo Expo, Montréal, Canada, May 2007, GT2007-27378.

[5] I. J. Day and C. Freeman, "Unstable behavior of low and highspeed compressors," Journal of Turbomachinery, vol. 116, no. 2, pp. 194-201, 1994.

[6] T. R. Camp and I. J. Day, "A study of spike and modal stall phenomena in a low-speed axial compressor," Journal of Turbomachinery, vol. 120, no. 3, pp. 393-401, 1998.

[7] I. J. Day, T. Breuer, J. Escuret, M. Cherrett, and A. Wilson, "Stall inception and the prospects for active control in four highspeed compressors," Journal of Turbomachinery, vol. 121, no. 1, pp. 18-27, 1999.

[8] M. M. Bright, H. K. Qammar, and L. Wang, "Investigation of pre-stall mode and pip inception in high-speed compressors through the use of correlation integral," Journal of Turbomachinery, vol. 121, no. 4, pp. 743-750, 1999.

[9] M. Inoue, M. Kuroumaru, S. Yoshida, and M. Furukawa, "Short and long length-scale disturbances leading to rotating stall in an axial compressor stage with different stator/rotor gaps," Journal of Turbomachinery, vol. 124, no. 3, pp. 376-384, 2002.

[10] J. Bergner, M. Kinzel, H.-P. Schiffer, and C. Hah, "Short lengthscale rotating stall inception in a transonic axial compressorexperimental investigation," in Proceedings of the 51st ASME Turbo Expo, pp. 131-140, Barcelona, Spain, May 2006, GT200690209.

[11] N. Gourdain, S. Burguburu, G. J. Michon, N. Ouayahya, F. Leboeuf, and S. Piot, "About the numerical simulation of rotating stall mechanisms in axial compressors," in Proceedings of the 51st ASME Turbo Expo, pp. 1723-1732, Barcelona, Spain, May 2006, GT2006-90223.

[12] I. Ariga, S. Masuda, and A. Ookita, "Inducer stall in a centrifugal compressor with the inlet distortion," ASME Journal of Turbomachinery, vol. 109, pp. 27-35, 1987.

[13] Y. Levy, J. Pismenny, A. Reissner, and W. Riess, "Experimental study of rotating stalls in a four-stage axial compressor," in Proceedings of the 47th International Gas Turbine and Aeroengine Congress and Exhibition, pp. 353-362, Amsterdam, The Netherlands, June 2002. 

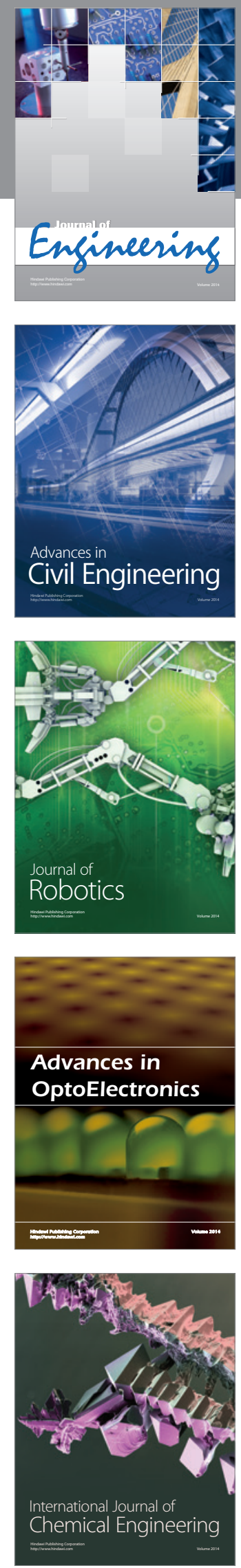

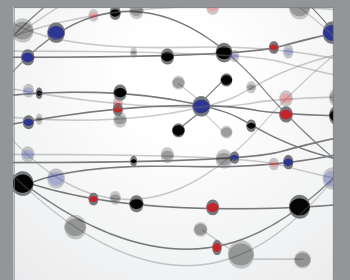

The Scientific World Journal
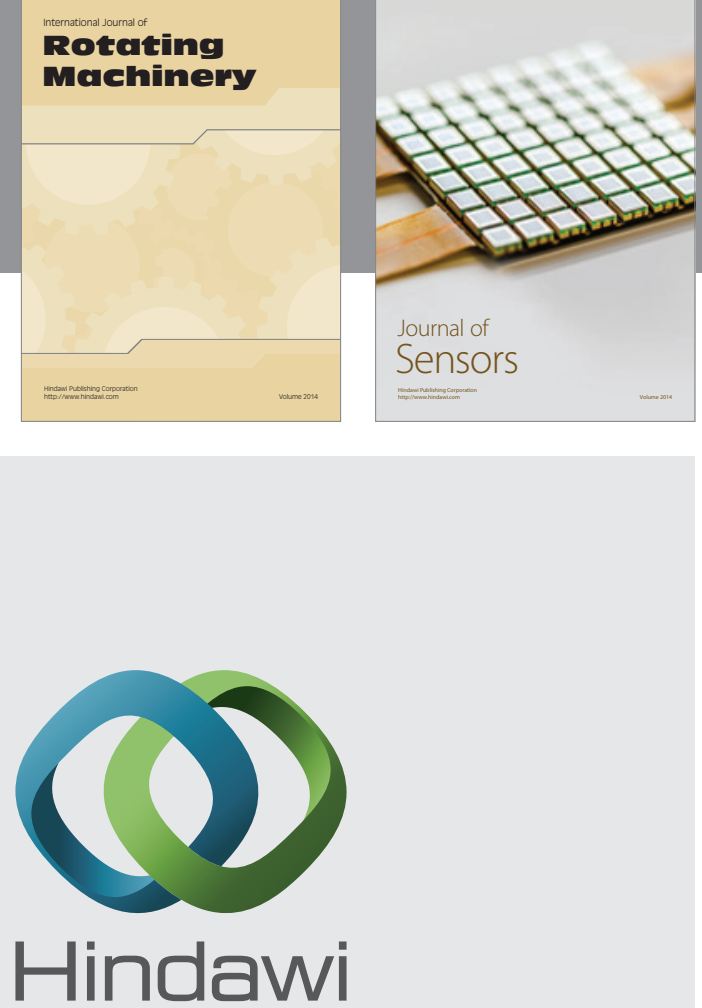

Submit your manuscripts at http://www.hindawi.com
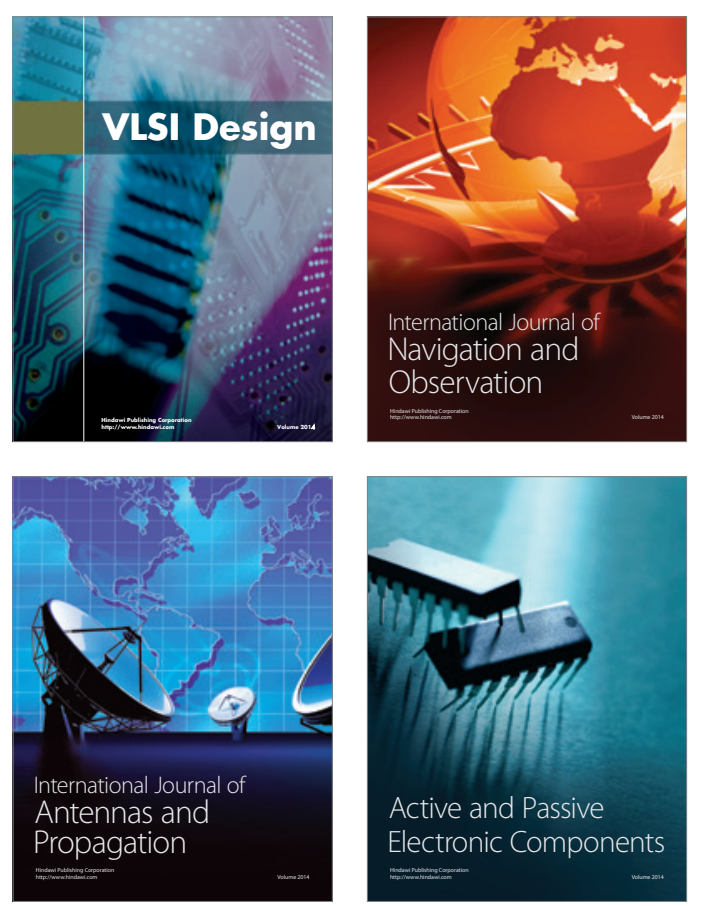
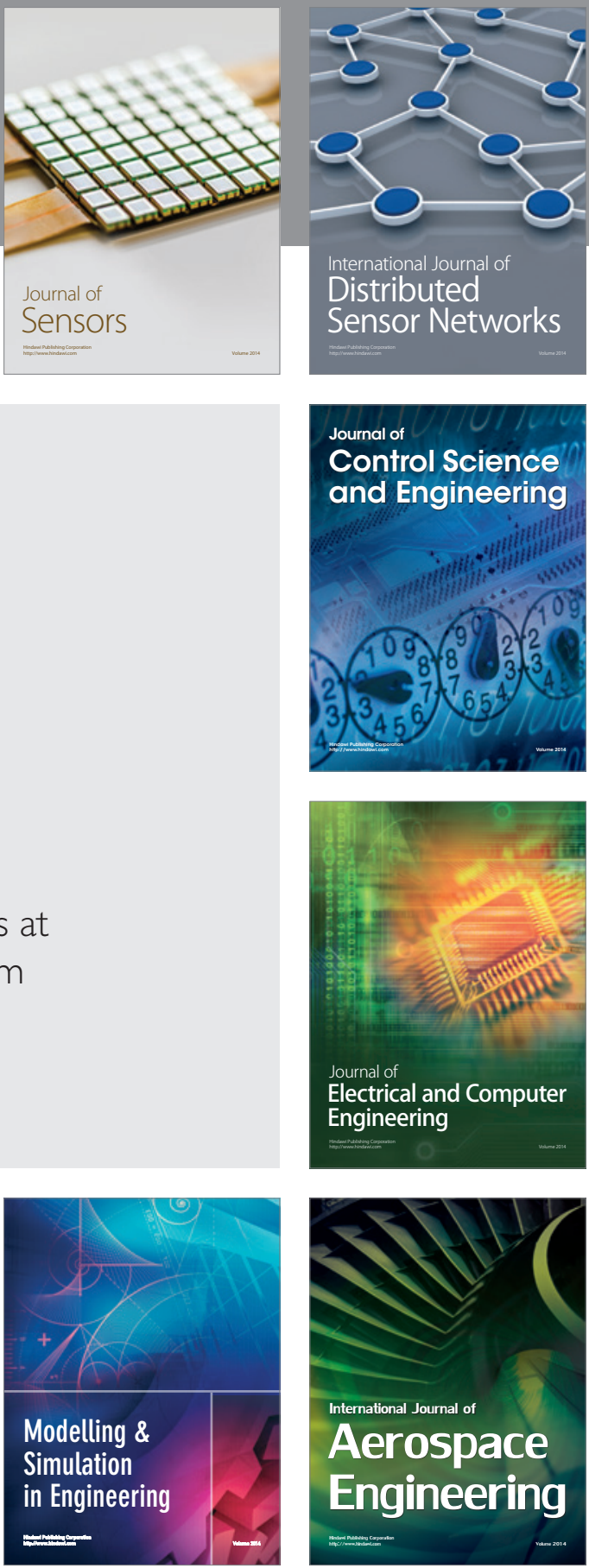

Journal of

Control Science

and Engineering
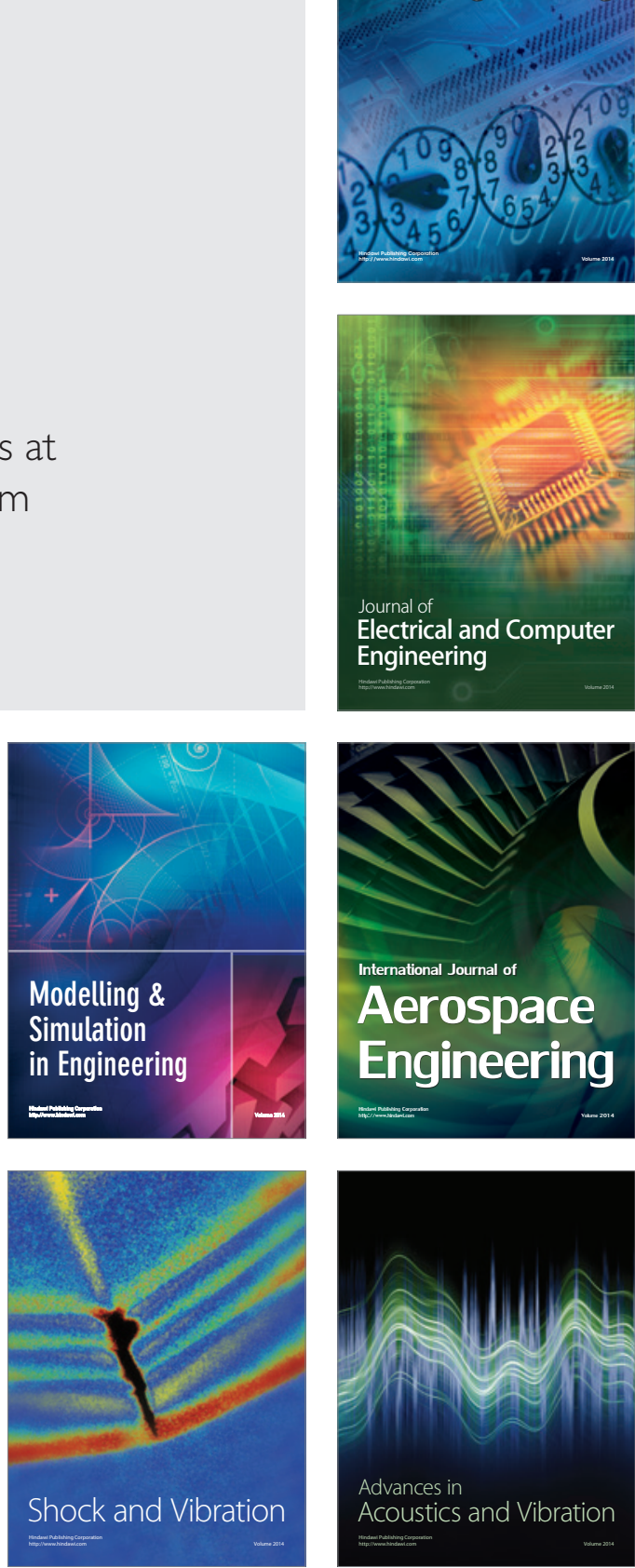